\title{
The Ground State Energy of a Classical Gas ${ }^{\star}$
}

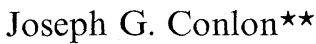

Institut für Theoretische Physik, Universität Wien, Boltzmanngasse 5, A-1090 Wien, Austria

\begin{abstract}
In this paper we study the ground state energy of a classical gas. Our interest centers mainly on Coulomb systems. We obtain some new lower bounds for the energy of a Coulomb gas. As a corollary of our results we can show that a fermionic system with relativistic kinetic energy and Coulomb interaction is stable. More precisely, let $H_{N}(\alpha)$ be the $N$ particle Hamiltonian$$
H_{N}(\alpha)=\alpha \sum_{i=1}^{N}\left(-\Delta_{i}\right)^{1 / 2}+\sum_{i<j}\left|x_{i}-x_{j}\right|^{-1}-\sum_{i, j}\left|x_{i}-R_{j}\right|^{-1}+\sum_{i<j}\left|R_{i}-R_{j}\right|^{-1},
$$

where $\Delta_{i}$ is the Laplacian in the variable $x_{i} \in \mathbb{R}^{3}$ and $R_{1}, \ldots, R_{N}$ are fixed points in $\mathbb{R}^{3}$. We show that for sufficiently large $\alpha$, independent of $N$, the Hamiltonian $H_{N}(\alpha)$ is nonnegative on the space of square integrable functions $\psi\left(x_{1}, \ldots, x_{N}\right)$, antisymmetric in the variables $x_{i}, 1 \leqq i \leqq N$.
\end{abstract}

\section{Introduction}

Consider the $N$ particle Hamiltonian $H_{N}^{R}(\alpha)$ defined by

$$
H_{N}^{R}(\alpha)=\alpha \sum_{i=1}^{N}\left(-\Delta_{i}\right)^{1 / 2}+\sum_{i<j}\left|x_{i}-x_{j}\right|^{-1}-\sum_{i, j}\left|x_{i}-R_{j}\right|^{-1}+\sum_{i<j}\left|R_{i}-R_{j}\right|^{-1},
$$

where $\Delta_{i}$ is the Laplacian in the variable $x_{i} \in \mathbb{R}^{3}$ and $R_{1}, \ldots, R_{N}$ are fixed points in $\mathbb{R}^{3}$. We prove the following:

Theorem 1.1. Let $H_{N}^{R}(\alpha)$ act on the space of square integrable functions $\psi\left(x_{1}, \ldots, x_{N}\right)$ on $\mathbb{R}^{3 N}$, antisymmetric in the variables $x_{i}, 1 \leqq i \leqq N$. Then there exists a universal constant $\alpha$ such that $H_{N}^{R}(\alpha) \geqq 0$.

\footnotetext{
* Research supported by grants from the Austrian National Science Foundation and University of Missouri research council

$\star \star$ Permanent address: Department of Mathematics, University of Missouri, Columbia, MO 65211, USA
} 
Theorem 1.1 may be regarded as the stability theorem for a relativistic system with Coulomb interactions. In our proof we require $\alpha \geqq 10^{200}$. This is clearly an absurdly high value for $\alpha$, and indeed for Theorem 1.1 to be relevant to physics the result should hold for some $\alpha<137$, [2]. Daubechies and Lieb [2] have proved a result analogous to Theorem 1.1 but with just one electron and many nuclei. In striking contrast to our theorem, their result holds for $\alpha \geqq \pi / 2$. Hence it seems quite possible that Theorem 1.1 does indeed hold for some $\alpha<137$.

The first problem of the type of Theorem 1.1 was considered by Dyson and Lenard, [3, 7]. Let $H_{N}^{C}(\alpha)$ be the Hamiltonian obtained from $H_{N}^{R}(\alpha)$ by replacing $\left(-\Delta_{i}\right)^{1 / 2}$ by $\left(-\Delta_{i}\right), 1 \leqq i \leqq N$. Dyson and Lenard showed that for every $\alpha>0$ there is a constant $C(\alpha)>0$ such that $H_{N}^{C}(\alpha)$ acting on the space of antisymmetric functions $\psi\left(x_{1}, \ldots, x_{N}\right)$ satisfies

$$
H_{N}^{C}(\alpha) \geqq-C(\alpha) N
$$

The Dyson-Lenard proof is very complicated and the constant $C(\alpha)$ correspondingly bad - of the order of $10^{14}$ when $\alpha=1$. In 1975 Lieb and Thirring $[10,16]$ produced a new proof of $(1.2)$ with a much better constant - of the order of 10 when $\alpha=1$. Their method was to bound $H_{N}^{C}(\alpha)$ below by the Thomas-Fermi Hamiltonian and then use the fact that the no binding theorem of Thomas-Fermi theory immediately implies stability of the Thomas-Fermi Hamiltonian. In 1975 Federbush [4] also obtained a new proof of (1.2). His approach is still quite complicated but, as was pointed out in [4], the advantage of his method is that it is more flexible than either the Dyson-Lenard approach or that of Lieb-Thirring. More recently Fefferman [5] has obtained some theorems converse to the DysonLenard theorem. He shows that in some sense a wave function which achieves the lower bound in (1.2) must be made up of about $N$ atoms.

In this paper we exploit the techniques developed by Federbush to prove Theorem 1.1. In fact the key Lemma 3.1 is taken from his paper [4]. We concentrate on obtaining good lower bounds on a classical Coulomb gas. Then Theorem 1.1 is obtained as a corollary of these results. We begin by considering the simplest of classical gases and eventually study the classical Coulomb gas with variable density. Our aim is to emphasize that our lower bounds on the Coulomb gas are merely a combination of some simple estimates on a gas with constant density together with certain localization procedures. As a final preliminary remark we note that Theorem 1.1.implies that (1.2) holds for all $\alpha>0$. The reason is that

$$
\alpha^{\prime} \sum_{i=1}^{N}\left(-\Delta_{i}\right) \geqq \alpha \sum_{i=1}^{N}\left(-\Delta_{i}\right)^{1 / 2}-\alpha^{2} N / 4 \alpha^{\prime}, \quad \alpha, \alpha^{\prime}>0 .
$$

This has been pointed out in [15].

We consider the ground state energy of a classical gas. Suppose the gas consists of $N$ particles in $\mathbb{R}^{3}$ interacting under a potential $\phi(x)$ which we assume is positive definite. The positions of the particles are described by a probability distribution function $P\left(x_{1}, \ldots, x_{N}\right)$ with one and two point functions $\varrho(x), \varrho(x, y)$ defined by

$$
\begin{gathered}
\varrho(x)=\sum_{i=1}^{N} \int P\left(x_{1}, \ldots, x_{i-1}, x, x_{i+1}, \ldots, x_{N}\right) d \hat{x}_{i}, \\
\varrho(x, y)=\sum_{i \neq j=1}^{N} \int P\left(x_{1}, \ldots, x_{i-1}, x, x_{i+1}, \ldots, x_{j-1}, y, x_{j+1}, x_{N}\right) d \hat{x}_{i, j} .
\end{gathered}
$$


The energy of the $N$ particles with distribution $P$ is then given by

$$
\mathscr{E}(P)=\frac{1}{2} \iint \varrho(x, y) \phi(x-y) d x d y .
$$

Now let us confine the $N$ particles to a cube $\Lambda$ in such a way that the density $N / \operatorname{Vol} \Lambda=\varrho$ is fixed. Let $E_{N}$ be the infimum of $\mathscr{E}(P)$ taken over all $P$ supported in $\Lambda^{N}$. Then we define the ground state energy of the gas at density, $\varrho, \mathscr{E}(\varrho)$, by

Our first result here is to show that

$$
\mathscr{E}(\varrho)=\liminf _{N \rightarrow \infty} E_{N} / N .
$$

$$
\lim _{\varrho \rightarrow \infty}\left[\mathscr{E}(\varrho)-\frac{1}{2} \varrho \int_{\mathbb{R} 3} \phi(x) d x\right]=-\frac{1}{2} \phi(0),
$$

for potentials $\phi \in L^{1} \cap L^{\infty}$. The identity (1.8) was essentially proved in Lewis et al. [8]. Here we prove it in a slightly different manner which relates to our subsequent work. Results of the genre of (1.8) were first obtained by Lieb [11].

Next we wish to consider a Coulomb gas so $\phi(x)=1 /|x|$. In this case the integral in (1.8) is infinite, so we must subtract it off by using equal numbers of positive and negative charges. Hence our Coulomb gas consists of $N$ negative particles described by a probability distribution $P_{1}\left(x_{1}, \ldots, x_{N}\right)$ and $N$ positive particles described by a probability distribution $P_{2}\left(x_{1}, \ldots, x_{N}\right)$. Thus in this gas the negative particles are independent of the positive. Let $\varrho_{1}(x), \varrho_{1}(x, y)$ be the one and two point functions corresponding to $P_{1}$ and $\varrho_{2}(x), \varrho_{2}(x, y)$ be the one and two point functions corresponding to $P_{2}$. Then the energy of the Coulomb gas is given by

$$
\mathscr{E}\left(P_{1}, P_{2}\right)=\frac{1}{2} \iint \frac{\varrho_{1}(x, y)}{|x-y|} d x d y+\frac{1}{2} \iint \frac{\varrho_{2}(x, y)}{|x-y|} d x d y-\iint \frac{\varrho_{1}(x) \varrho_{2}(y)}{|x-y|} d x d y .
$$

Now confine the $2 N$ particles to a cube $\Lambda$ with density $N / \operatorname{Vol} \Lambda=\varrho$ and assume $P_{2}$ is a constant so the positive particles are assumed to form a uniform background. Let $E_{N}$ be the infimum of $\mathscr{E}\left(P_{1}, P_{2}\right)$ taken over all $P_{1}$ supported in $\Lambda^{N}$. Then the ground state $\mathscr{E}(\varrho)$ for the Coulomb system is defined just as in (1.7). We shall show that

$$
\mathscr{E}(\varrho) \geqq-1.5 \varrho^{1 / 3} \text {. }
$$

The result (1.10) has already been obtained by Lieb and Narnhofer $[13,17]$ even with a slightly better constant (1.45). Our approach is rather different and generalizes to other singular potentials. The key property of the Coulomb potential seems to be that it can be written as a sum

$$
\phi(x)=\phi^{(1)}(x)+\phi^{(\infty)}(x),
$$

where the potential $\phi^{(1)}$ is pointwise positive and has $L^{1}$ norm $\left\|\phi^{(1)}\right\|_{1} \leqq \varepsilon^{2}$ for any arbitrary $\varepsilon>0$. The potential $\phi^{(\infty)}$ is positive definite and has $L^{\infty}$ norm $\left\|\phi^{(\infty)}\right\|_{\infty}$ $\leqq C / \varepsilon$, where $C$ is a constant independent of $\varepsilon$. Evidently any function $\phi(x)$ which has the property (1.11) must be both positive and positive definite and lie in the space $L_{w}^{3}$. It would be of interest to know if the converse were true.

It is not possible to prove (1.10) by using the fact that the first two integrals in (1.9) are positive and just bounding the third integral. In fact if we do this and assume $\varrho_{1}(x)=\varrho$ we get the bound

$$
E_{N} \geqq-C N^{5 / 3} \varrho^{1 / 3}
$$


Thus this approach gives a bound which is a factor $N^{2 / 3}$ larger than we want. For arbitrary distributions $P_{1}, P_{2}$, let us bound $\mathscr{E}\left(P_{1}, P_{2}\right)$ from below by using the positivity of the first two integrals of (1.9) and applying the weak Young inequality to the third. We wish also to exhibit the factor $N^{2 / 3}$ which should be the price we have paid for not taking into account the first two integrals of (1.9). We have the following:

Lemma 1.2. Let $p, q, \alpha, \beta$ satisfy the relations

$$
\begin{gathered}
\alpha+\beta=1, \quad p \alpha+q \beta=4 / 3, \\
p, q \geqq 1, \\
p \alpha \leqq 1, \quad q \beta \leqq 1,
\end{gathered}
$$

where if an equality holds in (1.14) then strict inequality must hold in (1.15). Then there is a constant $C_{p, q}$, depending only on $p, q$ such that

$$
\mathscr{E}\left(P_{1}, P_{2}\right) \geqq-C_{p, q} N^{2 / 3}\left\|\varrho_{1}\right\|_{p}^{p \alpha}\left\|\varrho_{2}\right\|_{q}^{q \beta} .
$$

Proof. Applying the weak Young inequality to the third integral of (1.9) we have

$$
\mathscr{E}\left(P_{1}, P_{2}\right) \geqq-C\left\|\varrho_{1}\right\|_{r}\left\|\varrho_{2}\right\|_{s},
$$

where $r$ and $s$ are related by

$$
\frac{1}{r}+\frac{1}{s}=\frac{5}{3}, \quad r, s>1
$$

Since the $L^{1}$ norm of $\varrho_{1}$ is $N$ we have by Hölder's inequality that

$$
\left\|\varrho_{1}\right\|_{r} \leqq N^{1 / r-\alpha}\left\|\varrho_{1}\right\|_{p}^{p \alpha}
$$

where

$$
\alpha=(r-1) /(p-1) r, \quad p \geqq r .
$$

Similarly we have

$$
\left\|\varrho_{2}\right\|_{s} \leqq N^{1 / s-\beta}\left\|\varrho_{2}\right\|_{q}^{q \beta}
$$

with

$$
\beta=(s-1) /(q-1) s, \quad q \geqq s .
$$

Suppose now that $p, q, \alpha, \beta$ satisfy the conditions of the theorem. Then we may define $r, s$ by (1.20) and (1.22). The condition (1.15) guarantees that $p \geqq r, q \geqq s$. Assume for the moment that strict inequality holds in (1.14). Hence $r, s>1$, and from (1.13) it follows that (1.18) holds. Thus (1.16) follows from (1.17), (1.19), and (1.21). Q.E.D.

In Sect. 3 we show that if we take into account the two positive integrals in (1.9) then just as in the simple case already discussed we may drop the factor $N^{2 / 3}$ in (1.16). Thus we have

Theorem 1.3. Let $p, q, \alpha, \beta$ satisfy the conditions of Lemma 1.2, and $C_{p, q}$ be the constant occurring in the lemma. Then there exist universal constants $C_{1}$ and $C_{2}$ such 
that

$$
\mathscr{E}\left(P_{1}, P_{2}\right) \geqq-\left[C_{1} C_{p, q}+C_{2}\right]\left\|\varrho_{1}\right\|_{p}^{p \alpha}\left\|\varrho_{2}\right\|_{q}^{q \beta} .
$$

In the appendix we obtain the values $C_{1}=10^{35}$ and $C_{2}=10^{162}$. These are clearly very bad values for the constants since two cases of Theorem 1.3 are already known with much better constants. The cases known are for $p=q=4 / 3$ with $P_{1}$ $=P_{2}$ and for $q=1$, which implies $p>3 / 2$. In the former case the result is Lieb's exchange energy inequality [9]. Lieb and Oxford [14] obtained the value 3.36 for the constant. In the latter the result is due to Lieb and Thirring $[10,16]$ and the constant is also of the order of 10 when $p=5 / 3$.

In Sect. 4 we obtain another lower bound on the Coulomb gas which is rather different from Theorem 1.3. It is given by

Theorem 1.4. There is a universal constant $C$ such that

$$
\mathscr{E}\left(P_{1}, P_{2}\right) \geqq-C\left[\int \sqrt{\varrho_{1}}(-\Delta)^{1 / 2} \sqrt{\varrho_{1}} d x+\int \varrho_{1}(x)^{4 / 3} d x\right] .
$$

The value for $C$ we obtain in the appendix is given by $C=10^{198}$. Theorem 1.4 is in a sense a version of Theorem 1.3 corresponding to the critical values $q=1$, $p=3 / 2$.

It is a short step from Theorem 1.4 to the proof of Theorem 1.1. Suppose the probability distribution $P_{1}$ is obtained from a fermion wave function $\psi$, so

$$
P_{1}\left(x_{1}, \ldots, x_{N}\right)=|\psi|^{2}\left(x_{1}, \ldots, x_{N}\right) .
$$

A theorem of Daubechies [1] tells us that the energy of a perfect fermion gas with wave function $\psi$ is bounded below by a constant times the second term on the right in (1.24). A second lemma tells us that the energy of this gas is also bounded below by a constant times the first term in (1.24). This lemma was proven for us by Barry Simon. The corresponding result for the Laplace operator was obtained by the Hoffmann-Ostenhofs [6]. Putting these two results together with Theorem 1.4 proves Theorem 1.1.

\section{The Case of Fixed Density}

We turn to the proof of (1.8). Our main lemma, which will also be of importance later is the following:

Lemma 2.1. Let $k(x, y)$ be a positive definite kernel and $P\left(x_{1}, \ldots, x_{N}\right)$ be an $N$ particle probability distribution with one and two point functions $\varrho(x), \varrho(x, y)$. Then

$$
\iint k(x, y) \varrho(x, y) d x d y \geqq \iint k(x, y) \varrho(x) \varrho(y) d x d y-\int k(x, x) \varrho(x) d x .
$$

Proof. Define numbers $e_{i}, 1 \leqq i \leqq 2 N$, by

$$
e_{i}=1, \quad 1 \leqq i \leqq N ; \quad e_{i}=-1, \quad N+1 \leqq i \leqq 2 N .
$$

Since $k(x, y)$ is positive definite we have

$$
\sum_{i, j=1}^{2 N} e_{i} e_{j} k\left(x_{i}, x_{j}\right) \geqq 0 .
$$


If we integrate (2.3) against the product $P\left(x_{1}, \ldots, x_{N}\right) P\left(x_{N+1}, \ldots, x_{2 N}\right)$ we obtain the inequality (2.1). Q.E.D.

Lemma 2.2. Let $\mathscr{E}(\varrho)$ be defined by (1.4). Then we have

$$
\mathscr{E}(\varrho) \geqq \frac{1}{2} \varrho \int \phi(x) d x-\frac{1}{2} \phi(0),
$$

provided the function $\phi(x)$ is positive definite and in $L^{1} \cap L^{\infty}$.

Proof. We take $k(x, y)=\phi(x-y)$ in Lemma 2.1. Thus we have

$$
\mathscr{E}(P) \geqq \frac{1}{2} \int_{\Lambda} \int_{\Lambda} \phi(x-y) \varrho(x) \varrho(y) d x d y-\frac{1}{2} N \phi(0) .
$$

Let $\Lambda_{h}$ be an $h$ neighbourhood of $\Lambda$, so

$$
\Lambda_{h}=\{x: d(x, \Lambda) \leqq h\} .
$$

The if $\chi_{\Lambda_{h}}$ is the characteristic function of $\Lambda_{h}$ we have by the positive definiteness of $\Lambda_{h}$ the inequality

$$
\begin{aligned}
& {\left[\iint_{\Lambda} \int_{\Lambda} \phi(x-y) \varrho(x) \varrho(y) d x d y\right]\left[\iint \phi(x-y) \chi_{\Lambda_{h}}(x) \chi_{\Lambda_{h}}(y) d x d y\right]} \\
& \quad \geqq\left[\iint \phi(x-y) \chi_{\Lambda_{h}}(x) \varrho(y) d x d y\right]^{2} .
\end{aligned}
$$

Hence from (2.5) we have that

$$
\mathscr{E}(P) \geqq \frac{1}{2}\left[\iint \phi(x-y) \chi_{\Lambda_{h}}(x) \varrho(y) d x d y\right]^{2} /\left[\iint \phi(x-y) \chi_{\Lambda_{h}}(x) \chi_{\Lambda_{h}}(y) d x d y\right]-\frac{1}{2} N \phi(0) \text {. }
$$

Now for any $\delta>0$ we can choose $h$ independent of $N, \Lambda$ such that for any $y \in \Lambda$

$$
\int_{\mathbb{R}^{3}} \phi(x) d x-\delta \leqq \int_{\Lambda_{h}} \phi(x-y) d x \leqq \int_{\mathbb{R}^{3}} \phi(x) d x+\delta .
$$

By choosing $\delta$ small enough in (2.9) we see from (2.8) that for any $\eta>0$ we have

$$
\mathscr{E}(\varrho) \geqq \frac{1}{2} \varrho \int \phi(x) d x-\frac{1}{2} \phi(0)-\eta \text {. }
$$

Letting $\eta \rightarrow 0$ proves the lemma. Q.E.D.

Lemma 2.3. With $\mathscr{E}(\varrho)$ as in (1.4) we have

$$
\limsup _{\varrho \rightarrow \infty}\left[\mathscr{E}(\varrho)-\frac{1}{2} \varrho \int \phi(x) d x\right] \leqq-\frac{1}{2} \phi(0) .
$$

Proof. Let $\psi_{1}(x), \ldots, \psi_{N}(x)$ be $N$ orthonormal wave functions and form the Hartree-Fock wave function

$$
\psi\left(x_{1}, \ldots, x_{N}\right)=\frac{1}{\sqrt{N !}} \operatorname{det} \psi_{i}\left(x_{j}\right) .
$$

Then if we take $P=|\psi|^{2}$ it is well known that the two point function $\varrho(x, y)$ is given by

$$
\varrho(x, y)=\varrho(x) \varrho(y)-\left|\sum_{i=1}^{N} \psi_{i}(x) \overline{\psi_{i}(y)}\right|^{2} .
$$

Now given the cube $\Lambda$ with $N / \operatorname{Vol} \Lambda=\varrho$, we divide $\Lambda$ into $N$ equal subcubes and define the $\psi_{i}(x)$ as constant functions supported on the subcubes. In this situation 
$\varrho(x)=\varrho$ and as $\varrho \rightarrow \infty$ the $\psi_{i}(x)$ are supported on increasingly smaller cubes. Thus, using the fact that $\phi(x)$ is continuous at $x=0$ we obtain the result (2.11). Q.E.D.

The previous three lemmas prove (1.5). The use of the Schwarz inequality in (2.7) is taken from Lewis et al. [8]. Next we state a lemma which appears trivial but is yet important.

Lemma 2.4. Suppose the potential $\phi(x)$ is pointwise positive. Then we have $\mathscr{E}(\varrho) \geqq 0$.

Observe that Lemma 2.2 applies for potentials which are positive definite while Lemma 2.4 applies for potentials which are pointwise positive. To prove (1.10) we use both of these lemmas by writing the Coulomb potential as a sum (1.11) of a positive potential and a positive definite potential. There are many ways of doing this. We choose the following:

$$
1 / 4 \pi|x|=\int_{0}^{\infty} \frac{1}{(4 \pi t)^{3 / 2}} \exp \left[-\frac{|x|^{2}}{4 t}\right] d t
$$

and so we have

$$
1 /|x|=\phi_{\lambda}^{(1)}(x)+\phi_{\lambda}^{(\infty)}(x)
$$

where

$$
\begin{aligned}
& \phi_{\lambda}^{(1)}(x)=4 \pi \int_{0}^{\lambda} \frac{1}{(4 \pi t)^{3 / 2}} \exp \left[-\frac{|x|^{2}}{4 t}\right] d t, \\
& \phi_{\lambda}^{(\infty)}(x)=4 \pi \int_{\lambda}^{\infty} \frac{1}{(4 \pi t)^{3 / 2}} \exp \left[-\frac{|x|^{2}}{4 t}\right] d t .
\end{aligned}
$$

Evidently we have

$$
\mathscr{E}(\varrho) \geqq \mathscr{E}_{\lambda}^{(1)}(\varrho)+\mathscr{E}_{\lambda}^{(\infty)}(\varrho),
$$

where $\mathscr{E}_{\lambda}^{(1)}(\varrho)$ is the ground state energy per particle corresponding to $\phi_{\lambda}^{(1)}$, and similar $\mathscr{E}_{\lambda}^{(\infty)}(\varrho)$ corresponds to $\phi_{\lambda}^{(\infty)}$. From Lemma 2.4 we have

$$
\mathscr{E}_{\lambda}^{(1)}(\varrho) \geqq-2 \pi \varrho \lambda,
$$

since we must take into account the attraction from the background charge. From Lemma 2.2 we have

$$
\mathscr{E}_{\lambda}^{(\infty)}(\varrho) \geqq-1 /(4 \pi \lambda)^{1 / 2}
$$

In (2.20) the infinite integral in (2.4) is subtracted off by means of the background charge. Thus we have

$$
\mathscr{E}(\varrho) \geqq-2 \pi \varrho \lambda-1 /(4 \pi \lambda)^{1 / 2}
$$

If we optimize (2.21) with respect to $\lambda$ for $\lambda>0$ we obtain (1.10).

It is easy to see from the above procedure how to generalize the result (1.10) to other singular potentials. Although the method employed in [13] to obtain (1.10) also uses a splitting similar to (1.11), this fact is not mentioned as the important principle involved. 


\section{The Coulomb Gas with Variable Density}

Here we turn to the proof of Theorem 1.3. The method is just like the proof of $(1.10)$ except that we need to vary the parameter $\lambda$ in (2.21) as $\varrho$ varies since $\lambda$ is proportional to $\varrho^{-2 / 3}$.

There are two important localization lemmas involved. The first is Lemma 2.1 and this lemma is already required in the proof of the exchange energy inequality [14]. The second localization lemma is needed in the case when $P_{1} \neq P_{2}$ and is taken from Federbush [4].

Lemma 3.1. Let $\chi_{i}(x), i \in \mathscr{L}$, denote the translates of an arbitrary $C^{\infty}$ function with compact support over the lattice $\mathscr{L}$ in $\mathbb{R}^{3}$. Then there is a constant $C>0$ such that the kernel

is positive definite.

$$
e^{-|x-y|}-C \sum_{i \in \mathscr{L}} \chi_{i}(x) \chi_{i}(y)
$$

Proof. The Fourier transform of $e^{-|x|}$ is a constant times $\left(1+k^{2}\right)^{-2}$. Now we have

$$
\iint \overline{f(x)} e^{-|x-y|} f(y) d x d y=\int|\hat{f}(k)|^{2}\left(1+k^{2}\right)^{-2} d k .
$$

Hence we need to prove that

$$
\int|\hat{f}(k)|^{2}\left(1+k^{2}\right)^{-2} d k \geqq C \sum_{i}\left|\left\langle\hat{f}, \hat{\chi}_{i}\right\rangle\right|_{2}=C \sum_{i}\left|\left\langle\frac{\hat{f}}{1+k^{2}},\left(1+k^{2}\right) \hat{\chi}_{i}\right\rangle\right|^{2} .
$$

Thus it is sufficient to show that for an arbitrary function $g(x)$ we have

$$
\int|g(x)|^{2} d x \geqq C \sum_{i}\left|\left\langle g,(-\Delta+1) \chi_{i}\right\rangle\right| \text {. }
$$

The inequality (3.4) however follows from the fact that the $\chi_{i}$ are translates of a function with compact support. Q.E.D.

Now let $f(x)$ be a nonnegative function to be chosen later and put

$$
k_{\infty}(x, y)=\int_{0}^{\infty} \chi[f(x)-u] \chi[f(y)-u] e^{-u|x-y|} d u,
$$

where $\chi(t)$ is the Heaviside function $\chi(t)=1$ if $t>0, \chi(t)=0$ if $t \leqq 0$. Observe that $k_{\infty}$ is analogous to $\phi^{(\infty)}$ in (2.17) except that instead of (2.14) we are using the representation

Let $k_{1}(x, y)$ be given by

$$
1 /|x|=\int_{0}^{\infty} e^{-u|x|} d u
$$

$$
k_{1}(x, y)=|x-y|^{-1}-k_{\infty}(x, y),
$$

so $k_{1}$ like $\phi^{(1)}$ in (2.14) is pointwise positive while $k_{\infty}$ like $\phi^{(\infty)}$ is positive definite. We apply Lemma 2.1 to obtain

$$
\begin{aligned}
\mathscr{E}\left(P_{1}, P_{2}\right) \geqq & \frac{1}{2} \iint k_{\infty}(x, y) \varrho_{1}(x) \varrho_{1}(y) d x d y \\
& -\iint \varrho_{1}(x) \varrho_{2}(y)\left[k_{\infty}(x, y)+k_{1}(x, y)\right] d x d y \\
& +\frac{1}{2} \iint k_{\infty}(x, y) \varrho_{2}(x) \varrho_{2}(y) d x d y-\frac{1}{2} \int k_{\infty}(x, x) \varrho_{1}(x) d x \\
& -\frac{1}{2} \int k_{\infty}(x, x) \varrho_{2}(x) d x \\
& =\frac{1}{2} \iint k_{\infty}(x, y)\left[\varrho_{1}(x)-\varrho_{2}(x)\right]\left[\varrho_{1}(y)-\varrho_{2}(y)\right] d x d y \\
& -\iint \varrho_{1}(x) \varrho_{2}(y) k_{1}(x, y) d x d y-\frac{1}{2} \int f(x)\left[\varrho_{1}(x)+\varrho_{2}(x)\right] d x .
\end{aligned}
$$


Hence if we use Lemma 3.1 we have

$$
\mathscr{E}\left(P_{1}, P_{2}\right) \geqq I_{1}-I_{2}-\frac{1}{2} I_{3}
$$

where

$$
\begin{gathered}
I_{1}=\frac{1}{2} C \iiint_{0}^{\infty} d u \chi[f(x)-u] \chi[f(y)-u] \\
\cdot \sum_{i \in \mathscr{L}} \chi_{i}(u x) \chi_{i}(u y)\left[\varrho_{1}(x)-\varrho_{2}(x)\right]\left[\varrho_{1}(y)-\varrho_{2}(y)\right] d x d y, \\
I_{2}=\iint \frac{\varrho_{1}(x) \varrho_{2}(y)}{|x-y|}\left[e^{-f(x)|x-y|}+e^{-f(y)|x-y|}\right] \mathrm{dxdy}, \\
I_{3}=\int f(x)\left[\varrho_{1}(x)+\varrho_{2}(x)\right] d x .
\end{gathered}
$$

We define the function $f(x)$. First we assume $N$ is large so that Theorem 1.3 is not just a corollary of Lemma 1.2. We put

$$
\varrho(x)=\varrho_{1}(x)+\varrho_{2}(x),
$$

and define $f(x)$ as the largest number $\lambda$ such that

$$
\int_{|x-y|<1 / \lambda} \varrho(y) d y \geqq 2 N_{0},
$$

where $N_{0}$ is a number to be fixed for all large $N$ later. Thus $f(x)$ is an average value of the one third power of the density at $x$.

We consider the sets

$$
E_{n}=\left\{x: 2^{n-1}<f(x) \leqq 2^{n}\right\}, \quad n=0, \pm 1, \ldots .
$$

For each $x \in E_{n}$ let $B_{x}$ be the ball with center $x$ and radius $2^{-(n+1)}$. Then it is evident that

$$
\begin{gathered}
\int_{B_{x}} \varrho(y) d y \leqq 2 N_{0}, \\
2^{n-2}<f(y) \leqq 2^{n+1}, \quad y \in B_{x} .
\end{gathered}
$$

The balls $B_{x}$ cover the neighbourhood of $E_{n}$ with radius $2^{-(n+2)}$ so we may choose a subcover which have finite intersection number smaller than some universal constant. Let us denote the subcover by $B_{n, i}, 1 \leqq i \leqq \kappa(n)$. We say that a ball $B=B_{n, i}$ belongs to the set $\mathscr{S}$ if

$$
\int_{B} \varrho(y) d y \geqq 2 N_{0} / C_{0},
$$

where $C_{0}$ is a universal constant to be given in the following lemma.

Lemma 3.2. There is a universal constant $C_{0}$ and a constant $\kappa$ such that

$$
I_{3} \leqq \kappa \sum_{B \in \mathscr{S}} \int_{B} f(y) \varrho(y) d y .
$$

Proof. Evidently we have

$$
I_{3} \leqq \sum_{n=-\infty}^{\infty} \sum_{i=1}^{\kappa(n)} 2^{n+1} \int_{B_{n, 1}} \varrho(y) d y .
$$


For the ball $B_{n, i}$, let $B_{n, i}^{*}$ be the ball concentric with $B_{n, i}$ but with radius $1 / 2^{n-1}$. Then from (3.14) we have

$$
\int_{B_{n}^{*}, i} \varrho(y) d y \geqq 2 N_{0} .
$$

Observe that

$$
f(y)>2^{n-2}, \quad y \in B_{n, i}^{*} .
$$

Since the sets $B_{n, i}$ have finite intersection number, $1 \leqq i \leqq \kappa(n)$, so also do the sets $B_{n, i}^{*}, 1 \leqq i \leqq \kappa(n)$. Let the intersection number of the $B_{n, i}^{*}$ be smaller than $2^{\gamma-1}$, where $\gamma$ is some universal constant, $\gamma \geqq 1$. Then we may choose a universal constant $C_{0}$ such that one of two possibilities holds: Either there is a set $B_{n-1, j}, B_{n, j}, \ldots, B_{n+\gamma, j}$ in $\mathscr{S}$ which intersects $B_{n, i}^{*}$, or the integral of $\varrho(y)$ over the set

$$
B_{n, i}^{*} \cap \bigcup_{m=n+\gamma+1}^{\infty} \bigcup_{j=1}^{\kappa(m)} B_{m, j}
$$

is larger than the integral of $\varrho(y)$ over $B_{n, i}$.

We therefore conclude that

$$
\begin{aligned}
\sum_{i=1}^{\kappa(n)} \int_{\boldsymbol{B}_{n, 2}} \varrho(y) d y \leqq & A \sum_{m=n-1}^{n+\gamma} \sum_{\boldsymbol{B}_{m, j} \in \mathscr{S}} \int_{\boldsymbol{B}_{m, j}} \varrho(y) d y \\
& +2^{\gamma-1} \sum_{m=n+\gamma+1}^{\infty} \sum_{j=1}^{\kappa(m)} \int_{B_{m, j}} \varrho(y) d y,
\end{aligned}
$$

where $A$ is a universal constant depending on $\gamma$.

Now if we multiply (3.24) by $2^{n+1}$ and sum with respect to $n$ we have-denoting by $I$ the right side of $(3.20)$ - the inequality

$$
I \leqq 16 A(\gamma+1) \sum_{B \in \mathscr{S}} \int_{B} f(y) \varrho(y) d y+\frac{1}{2} I .
$$

The result follows from (3.25). Q.E.D.

Lemma 3.3. Let $p, q, \alpha, \beta$ satisfy the conditions of Lemma 1.2. Then there is a constant $C\left(N_{0}\right)$ depending on $N_{0}$ and a universal constant $\kappa$ such that

$$
I_{2} \leqq C\left(N_{0}\right)\left\|\varrho_{1}\right\|_{p}^{p \alpha}\left\|\varrho_{2}\right\|_{q}^{q \beta}+\kappa \sum_{B \in \mathscr{S}} \int_{B} f(y) \varrho(y) d y .
$$

Proof. We write the integral

$$
\iint \frac{\varrho_{1}(x) \varrho_{2}(y)}{|x-y|} e^{-f(x)|x-y|} d x d y=\iiint_{|x-y|<1 / 8 f(x)}+\iiint_{|x-y| \geqq 1 / 8 f(x)} .
$$

Since the sets $B_{n, i}$ cover a neighbourhood of $E_{n}$ with radius $2^{-(n+2)}$ and for $x \in E_{n}$ we have $f(x)>2^{n-1}$, it follows that the integral in (3.27) over $|x-y|<1 / 8 f(x)$ is bounded by

$$
\sum_{n=-\infty}^{\infty} \sum_{i=1}^{\kappa(n)} \int_{B_{n, i}} \int_{B_{n, i}} \frac{\varrho_{1}(x) \varrho_{2}(y)}{|x-y|} d x d y .
$$

Since the sets $B_{n, i},-\infty<n<\infty, 1 \leqq i \leqq \kappa(n)$, have finite intersection number we can apply the Young inequality to each term in (3.28) just as in Lemma 1.2, using the 
fact that

$$
\int_{B_{n, i}} \varrho(y) d y \leqq 2 N_{0} .
$$

Then a further application of the Hölder inequality with exponents $1 / \alpha, 1 / \beta$ yields the first term in the right side of (3.26) as a bound on (3.28).

To deal with the second integral in (3.27) we define for integers $r=1,2, \ldots$,

$$
a_{r}=\int f(x) \varrho_{1}(x) \int_{|x-y|<r / f(x)} \varrho_{2}(y) d y .
$$

Evidently the second integral is bounded by

$$
7 a_{1}+\sum_{r=1}^{\infty} a_{r} e^{-r+1} .
$$

Let $U_{n, i}(r), 1 \leqq i \leqq \kappa(n)$, be the ball concentric with $B_{n, i}$ but with radius $(r+1) / 2^{n-1}$. Then, in view of (3.15), we have that

$$
a_{r} \leqq \sum_{n=-\infty}^{\infty} 2^{n+1} \sum_{i=1}^{\kappa(n)} \int_{B_{n, i}} \varrho_{1}(x) d x \int_{U_{n, i}(r)} \varrho_{2}(y) d y .
$$

Next, observe that the sets $U_{n, i}(r), 1 \leqq i \leqq \kappa(n)$, have finite intersection number smaller than a universal constant times $r^{3}$ and that $U_{n, i}$ can only intersect $E_{j}$ for $j$ which satisfy

$$
2^{j}>2^{n-1} /(r+2)
$$

Let the set $S_{n, i, k}$ be defined by

$$
S_{n, i, k}=\left\{j: B_{k, j} \cap U_{n, i} \neq \emptyset\right\},
$$

and $R$ be the smallest integer such that

$$
2^{R-1} \geqq r+2 .
$$

Then it follows from (3.33) and (3.17) that if

$$
k<n-R \text {, }
$$

then $S_{n, i, k}$ is empty. Furthermore, by the finite intersection property of the sets $U_{n, i}(r), 1 \leqq i \leqq \kappa(n)$, it follows that any integer $j, 1 \leqq j \leqq \kappa(k)$, occurs at most a universal constant times $r^{3}$ in the disjoint union

$$
\bigcup_{i=1}^{k(n)} S_{n, i, k}
$$

From (3.36) we have that

$$
\int_{U_{n, \imath}(r)} \varrho_{2}(y) d y \leqq \sum_{k=n-R}^{\infty} \sum_{j \in S_{n, i, k}} \int_{B_{k, j}} \varrho_{2}(y) d y .
$$

Hence for any integer $m_{0}$ we have

$$
\begin{aligned}
a_{r} \leqq & \sum_{t=-R}^{m_{0}-1} \sum_{n=-\infty}^{\infty} 2^{n+1} \sum_{i=1}^{\kappa(n)} \int_{B_{n, i}} \varrho_{1}(x) d x \sum_{j \in S_{n, i, n+t} B_{n+t, j}} \varrho_{2}(y) d y \\
& +\sum_{n=-\infty}^{\infty} \sum_{i=1}^{\kappa(n)} 2^{n+2} N_{0} \sum_{k=m_{0}+n} \sum_{j \in S_{n, i, k}} \int_{B_{k, j}} \varrho(y) d y,
\end{aligned}
$$


where in the second sum we have used (3.29). From (3.37) we see that the second sum in (3.39) is bounded by

$$
c r^{3} N_{0} 2^{-m_{0}} \sum_{k=-\infty}^{\infty} \sum_{j=1}^{\kappa(k)} 2^{k+1} \int_{B_{k, j}} \varrho(y) d y,
$$

where $c$ is a universal constant. Now from Lemma 3.2 we have that (3.40) is bounded by

$$
\kappa c r^{3} N_{0} 2^{-m_{0}} \sum_{B \in \mathscr{S}} \int_{B} f(y) \varrho(y) d y .
$$

Next we show that

$$
\begin{gathered}
\sum_{n=-\infty}^{\infty} 2^{n+1} \sum_{i=1}^{\kappa(n)} \int_{B_{n, l}} \varrho_{1}(x) d x \sum_{j \in S_{n, i, n+t} B_{n+t, j}} \varrho_{2}(y) d y \\
\leqq C\left(N_{0}\right) r^{3} 2^{3(1-q \beta) t}\left\|\varrho_{1}\right\|_{p}^{p \alpha}\left\|\varrho_{2}\right\|_{q}^{q \beta},
\end{gathered}
$$

where the constant $C\left(N_{0}\right)$ depends only on $N_{0}$. To do this first observe that the cardinality of $S_{n, i, n+t}$ is bounded as

$$
\left|S_{n, i, n+t}\right| \leqq c 2^{3 t} r^{3},
$$

for some universal constant $c$. Now the sum in (3.42) is bounded by

$$
\begin{aligned}
& C\left(N_{0}\right) \sum_{n=-\infty}^{\infty} 2^{n+1} \sum_{i=1}^{\kappa(n)}\left[\int_{B_{n, i}} \varrho_{1}(x) d x\right]^{p \alpha} \sum_{j \in S_{n, i, n+t}}\left[\int_{B_{n+t, j}} \varrho_{2}(y) d y\right]^{q \beta} \\
& \leqq C\left(N_{0}\right) 2^{-3 \beta(q-1) t} \sum_{n=-\infty}^{\infty} \sum_{i=1}^{\kappa(n)}\left[\int_{B_{n, t}} \varrho_{1}(x)^{p} d x\right]^{\alpha} \sum_{j \in S_{n, i, n+t}}\left[\int_{B_{n+t, j}} \varrho_{2}(y)^{q} d y\right]^{\beta} \\
& \leqq C\left(N_{0}\right) 2^{-3 \beta(q-1) t}\left[\sum_{n=-\infty}^{\infty} \sum_{i=1}^{\kappa(n)} \sum_{j \in S_{n, i, n+t}} \int_{B_{n, i}} \varrho_{1}(x)^{p} d x\right]^{\alpha} \\
& \cdot\left[\sum_{n=-\infty}^{\infty} \sum_{i=1}^{\kappa(n)} \sum_{j \in S_{n, i, n+t} B_{n+t, j}} \varrho_{2}(y)^{q} d y\right]^{\beta} \\
& \leqq C\left(N_{0}\right) r^{3} 2^{3(1-q \beta) t}\left\|\varrho_{1}\right\|_{p}^{p \alpha}\left\|\varrho_{2}\right\|_{q}^{q \beta} .
\end{aligned}
$$

Now if we choose $m_{0}$ such that

$$
N_{0} 2^{-m_{0}} \leqq 1
$$

we can bound $a_{r}$ as in (3.26). Then summing with respect to $r$ as in (3.31) yields the result. Q.E.D.

Lemma 3.4. Given a constant $\kappa$ we can choose the number $N_{0}$ such that

$$
I_{1}-\kappa \sum_{B \in \mathscr{S}} \int_{B} f(y) \varrho(y) d y \geqq-C\left(N_{0}\right)\left\|\varrho_{1}\right\|_{p}^{p \alpha}\left\|\varrho_{2}\right\|_{q}^{q \beta} .
$$

Proof. We first define the lattice $\mathscr{L}$ of Lemma 3.1. A vector $i \in \mathscr{L}$ if $128 i$ is a vector with integer components. Thus any point $x \in \mathbb{R}^{3}$ is less than a distance $1 / 128$ from some point of $\mathscr{L}$. Let $\phi(x)$ be a $C^{\infty}$ function which is such that $0 \leqq \phi(x) \leqq 1, x \in \mathbb{R}^{3}$, and

$$
\phi(x)=1 \quad \text { if } \quad|x| \leqq 9 / 64 ; \quad \phi(x)=0 \quad \text { if }|x| \geqq 5 / 32 .
$$

Then the functions $\chi_{i}(x)$ are translates of $\phi(x)$ through the lattice $\mathscr{L}$. 
We write $I_{1}$ in (3.10) as

$$
I_{1}=\sum_{n=-\infty}^{\infty} \int_{2^{n-3}}^{2^{n-2}} d u
$$

Next consider a ball $B_{n, k}, 1 \leqq k \leqq \kappa(n)$, which has radius $1 / 2^{n+1}$. Then there is a vector $i \in \mathscr{L}$ such that $i / 2^{n-2}$ is less than $1 / 2^{n+5}$ from the center of $B_{n, k}$. Furthermore

$$
\chi_{i}\left(2^{n-2} x\right)=\phi\left(2^{n-2} x-i\right)=\phi\left(2^{n-2}\left[x-i / 2^{n-2}\right]\right) .
$$

Thus $\chi_{i}\left(2^{n-2} x\right)=1$ if $x \in B_{n, k}$ and $\chi_{i}\left(2^{n-2} x\right)=0$ if $x \notin B_{n, k}^{*}$, when $B_{n, k}^{*}$ is the ball concentric with $B_{n, k}$ but with radius $3 / 2^{n+2}$. One can also easily see that if

$$
2^{n-2} \geqq u \geqq\left(\frac{1}{4}-\frac{1}{128}\right) 2^{n},
$$

then there is an $i \in \mathscr{L}$ - which may vary with $u$-such that $\chi_{i}(u x)=1$ if $x \in B_{n, k}$ and $\chi_{i}(u x)=0$ if $x \notin B_{n, k}^{*}$.

Now suppose $B=B_{n, k} \in \mathscr{S}$. Then, in view of (3.18) we can assume without loss of generality that

$$
\int_{B} \varrho_{1}(y) d y \geqq N_{0} / C_{0} .
$$

If we also have

$$
\int_{B_{n, k}^{*}} \varrho_{2}(y) d y \leqq N_{0} / 2 C_{0},
$$

we shall say $B \in \mathscr{S}^{\prime}$. If we fix $n$ and let $k$ vary, $1 \leqq k \leqq \kappa(n)$, then it is clear by the finite intersection property of the $B_{n, k}^{*}$ that any $i \in \mathscr{L}$ which occurs with $\chi_{i}(u x)=1$ for $x \in B_{n, k}$, occurs only finitely many times. We therefore conclude that for $u$ satisfying (3.50) there is a universal constant $C$ such that

$$
\begin{gathered}
\iint \chi[f(x)-u] \chi[f(y)-u] \sum_{i \in \mathscr{L}} \chi_{i}(u x) \chi_{i}(u y) \\
\cdot\left[\varrho_{1}(x)-\varrho_{2}(x)\right]\left[\varrho_{1}(y)-\varrho_{2}(y)\right] d x d y \\
\geqq c N_{0}^{2} \#\left\{B=B_{n, k} \in \mathscr{S}^{\prime}\right\} .
\end{gathered}
$$

Now if we use (3.48) and choose $N_{0}$ large enough we may conclude from (3.29) that

$$
I_{1} \geqq \kappa \sum_{B \in \mathscr{S}^{\prime}} \int_{B} f(y) \varrho(y) d y .
$$

It remains for us to deal with $B \notin \mathscr{S}^{\prime}$. In that case we see that

$$
\begin{aligned}
\sum_{B \in \mathscr{S} \backslash \mathscr{S}^{\prime}} \int_{B} f(y) \varrho(y) d y & \leqq \sum_{B=B_{n, k} \in \mathscr{S} \backslash \mathscr{S}^{\prime}} 2^{n+1} \int_{B_{n, k}^{*}} \varrho(y) d y \\
& \leqq C\left(N_{0}\right) \sum_{n=-\infty}^{\infty} \sum_{k=1}^{\kappa(n)} 2^{n+1}\left[\int_{B_{n, k}^{*}} \varrho_{1}(y) d y\right]^{p \alpha}\left[\int_{B_{n, k}^{*}} \varrho_{2}(y) d y\right]^{q \beta},
\end{aligned}
$$

for some constant $C\left(N_{0}\right)$ depending on $N_{0}$. Now we can estimate the last sum in (3.55) just as before to obtain (3.46). Q.E.D.

Finally we observe that Theorem 1.3 follows from (3.9) and the previous lemmas. 


\section{Stability of the Relativistic System}

We turn to the proof of Theorem 1.4. We define the operator $H_{1}$ to be

$$
H_{1}=(-\Delta)^{1 / 2} \text {, }
$$

and assume that $\varrho_{1}(x)$ is such that $\varrho_{1}(x)^{1 / 2}$ is in the domain of $H_{1}$. First we show that

$$
\begin{aligned}
I_{2} \leqq & C\left(N_{0}\right)\left[\int_{\mathbb{R}^{3}} \varrho_{1}(x)^{4 / 3} d x+\left\langle\varrho_{1}^{1 / 2}, H_{1} \varrho_{1}^{1 / 2}\right\rangle\right] \\
& +\kappa \sum_{B \in \mathscr{S}} \int_{B} f(y) \varrho(y) d y .
\end{aligned}
$$

To show (4.2) we must estimate (3.42) somewhat differently. We divide the sets $B=B_{n, i}$ into two kinds. We say $B \in \mathscr{A}$ if

$$
\int_{B} \varrho_{1}(x) d x \geqq 1
$$

We write (3.42) as

$$
\sum_{B \in \mathscr{A}}+\sum_{B \notin \mathscr{A}}
$$

Then we see that

$$
\begin{aligned}
\sum_{B \in \mathscr{A}} & \leqq C\left(N_{0}\right) 2^{3 t} r^{3} \sum_{n=-\infty}^{\infty} 2^{n} \sum_{i=1}^{\kappa(n)} \sum_{B_{n, i} \in \mathscr{A}} \int_{B_{n, i}} \varrho_{1}(x) d x \\
& \leqq C\left(N_{0}\right) 2^{3 t} r^{3} \sum_{n=-\infty}^{\infty} 2^{n} \sum_{i=1}^{\kappa(n)} \sum_{B_{n, i} \in \mathscr{A}}\left[\int_{B_{n, i}} \varrho_{1}(x) d x\right]^{4 / 3} \\
& \leqq C\left(N_{0}\right) 2^{3 t} r^{3} \int_{\mathbb{R}^{3}} \varrho_{1}(x)^{4 / 3} d x,
\end{aligned}
$$

on using Hölder's inequality and the finite intersection property of the balls $B_{n, i}$. We have also

$$
\begin{aligned}
\sum_{B \notin \mathscr{A}} & \leqq \sum_{n=-\infty}^{\infty} \sum_{i=1}^{\kappa(n)} \sum_{j \in S_{n, i, n+t}} 2^{n} \int_{B_{n+t, j}} \varrho_{2}(y) d y \\
& \leqq c 2^{-t} r^{3} \sum_{n=-\infty}^{\infty} \sum_{i=1}^{\kappa(n)} 2^{n+1} \int_{B_{n, i}} \varrho(y) d y
\end{aligned}
$$

where $c$ is a universal constant. Hence, on using Lemma 3.2, we have that

$$
a_{r} \leqq C\left(N_{0}\right) r^{3} \int_{\mathbb{R}^{3}} \varrho_{1}(x)^{4 / 3} d x+\kappa r^{4} \sum_{B \in \mathscr{S}} \int_{B} f(y) \varrho(y) d y,
$$

where $\kappa$ is a constant independent of $N_{0}$. Thus on summing (3.31) we shall get the estimate (4.2) provided we can deal with (3.28).

To do this we use the Fefferman-Phong technique [5]. Let $Q_{0}$ be a large cube in $\mathbb{R}^{3}$ and make a dyadic decomposition of $Q_{0}$. For a function $u \in L^{2}\left(Q_{0}\right)$ and a dyadic subcube $Q$ of $Q_{0}$, let $\hat{u}(Q)$ be the projection of $u$ onto the functions which are linear + constant on the 8 subcubes of $Q$ and orthogonal to the functions linear + constant on all of $Q$. Then if $u \in L^{2}\left(Q_{0}\right)$ is orthogonal to functions linear + constant on all of $Q_{0}$ we have

$$
u=\sum_{Q \subset Q_{0}} \hat{u}(Q) .
$$


Now from [5] we see that if $u$ is in the domain of $H_{1}$ then there is a constant $C$ such that

$$
\left\langle u, H_{1} u\right\rangle \geqq C \sum_{Q \subset Q_{0}}(\operatorname{diam} Q)^{-1}\|\hat{u}(Q)\|^{2} .
$$

For a ball $B_{n, i}$ - with radius $1 / 2^{n+1}-$ let $B_{n, i}^{*}$ be the ball concentric with $B_{n, i}$ but with radius $3 / 2^{n+2}$, so the balls $B_{n, i}^{*}$ have the finite intersection property. Suppose $B_{n, i}$ is contained in $Q_{0}$. Then $B_{n, i}$ is covered by a finite number of dyadic subcubes of $Q_{0}$, each with the same diameter $\sim 2^{-n}$ and contained in $B_{n, i}^{*}$. Let $Q$ be one of these subcubes and $P_{Q}$ be the projection operator onto linear + constant on $Q$. Then we know from [5] that

$$
\int_{Q}\left[\sqrt{\varrho_{1}}(x)-P_{Q} \sqrt{\varrho_{1}}(x)\right]^{2} \frac{d x}{|x-y|} \leqq C \sum_{Q^{\prime} \subset Q}\left(\operatorname{diam} Q^{\prime}\right)^{-1}\left\|\sqrt{\varrho_{1}}\left(Q^{\prime}\right)\right\|^{2},
$$

for some constant $C$ independent of $y$ and $Q$.

It is easy to see that

$$
\int_{Q}\left[P_{Q} \sqrt{\varrho_{1}}(x)\right]^{2} \frac{d x}{|x-y|} \leqq C(\operatorname{diam} Q)^{-1} \int_{Q} \varrho_{1}(x) d x,
$$

with $C$ independent of $Q$. Hence if $B_{n, i}$ is covered by cubes $Q_{1}, \ldots, Q_{L}$, we conclude that

$$
\begin{aligned}
& \int_{B_{n, i}} \int_{B_{n, 2}} \frac{\varrho_{1}(x) \varrho_{2}(y)}{|x-y|} d x d y \leqq C 2^{n} \int_{B_{n, i}^{*}} \varrho_{1}(x) d x \int_{B_{n, i}^{*}} \varrho_{2}(y) d y \\
& \quad+C\left(N_{0}\right) \sum_{j=1}^{L} \sum_{Q^{\prime} \subset Q_{j}}\left(\operatorname{diam} Q^{\prime}\right)^{-1}\left\|\sqrt{\varrho_{1}}\left(Q^{\prime}\right)\right\|^{2},
\end{aligned}
$$

where here we have used (3.29).

Next we sum (4.12) with respect to $n, i$ with $B_{n, i} \subset Q_{0}$. We may estimate the first sum just as was done in (4.7). To estimate the second sum in (4.12) observe that by the finite intersection property of the sets $B_{n, i}^{*}$ that any dyadic cube $Q$ we have chosen to cover $B_{n, i}$ occurs only a finite number of times as we vary $n, i$. The result (4.2) then follows from (4.9).

To complete the proof of the theorem we shall replace the right side of the inequality $(3.46)$ by

$$
-C\left(N_{0}\right) \int_{\mathbb{R}^{3}} \varrho_{1}(x)^{4 / 3} d x .
$$

We easily observe that, instead of the estimate (3.55) we have

$$
\begin{aligned}
\sum_{B \in \mathscr{S} \backslash \mathscr{S}^{\prime}} \int_{B} f(y) \varrho(y) d y & \leqq C\left(N_{0}\right) \sum_{B=B_{n, k}} 2^{n}\left[\int_{B} \varrho_{1}(y) d y\right]^{4 / 3} \\
& \leqq C\left(N_{0}\right) \int_{\mathbb{R}^{3}} \varrho_{1}(x)^{4 / 3} d x . \quad \text { Q.E.D. }
\end{aligned}
$$

Finally we complete the proof of Theorem 1.1 by bounding below a fermion gas in terms of the density. Let $H_{N}$ be the $N$ particle Hamiltonian

$$
H_{N}=\sum_{i=1}^{N}\left(-\Delta_{i}\right)^{1 / 2}
$$


acting on wave functions $\psi\left(x_{1}, \ldots, x_{N}\right)$. Here $\Delta_{i}$ is the Laplacian in the $x_{i}$ variable. The following lemma is due to Daubechies [1].

Lemma 4.1. Let $\psi$ be a normalized Fermion wave function in the domain of $H_{N}$ and with one point function $\varrho(x)$. Then there is a constant $C$ independent of $N$ such that

$$
\left\langle\psi, H_{N} \psi\right\rangle \geqq C \int_{\mathbb{R}^{3}} \varrho(x)^{4 / 3} d x .
$$

We are indebted to Barry Simon for the proof of the following lemma. The corresponding result for the Laplace operator has been proved by the HoffmannOstenhofs [6].

Lemma 4.2. Let $\psi$ be a normalized wave function in the domain of $H_{N}$ and with one point function $\varrho(x)$. Then

$$
\left\langle\psi, H_{N} \psi\right\rangle \geqq\left\langle\varrho^{1 / 2}, H_{1} \varrho^{1 / 2}\right\rangle .
$$

Proof. Let the kernel $k(x, y)$ be defined by

$$
k(x, y)=\sum_{i=1}^{N} \int \bar{\psi}\left(x_{1}, \ldots, x_{i-1}, x, x_{i+1}, \ldots, x_{N}\right) \psi\left(x_{1}, \ldots, x_{i-1}, y, x_{i+1}, \ldots, x_{N}\right) d \hat{x}_{i} .
$$

Then it is easy to see that $k(x, y)$ is a positive definite kernel and

$$
k(x, x)=\varrho(x) .
$$

Since $k$ is positive definite we have from Schwarz's inequality,

$$
|k(x, y)| \leqq k(x, x)^{1 / 2} k(y, y)^{1 / 2} \text {. }
$$

The key observation is that the operator $\exp \left(-H_{1} t\right)$ has positive integral kernel

Thus if we put

$$
e^{-H_{1} t}(x, y)
$$

$$
F_{N}(t)=\sum_{i=1}^{N}\left\langle\psi, \exp \left(-\left(-\Delta_{i}\right)^{1 / 2} t\right) \psi\right\rangle
$$

and let

$$
F(t)=\left\langle\varrho^{1 / 2}, \exp \left(-H_{1} t\right) \varrho^{1 / 2}\right\rangle
$$

we see that

$$
F_{N}(0)=F(0)=N \text {. }
$$

Now for $t>0$ we have from (4.20),

$$
\begin{aligned}
F_{N}(t) & =\iint e^{-H_{1} t}(x, y) k(x, y) d x d y \\
& \leqq \iint e^{-H_{1} t}(x, y) k(x, x)^{1 / 2} k(y, y)^{1 / 2} d x d y=F(t) .
\end{aligned}
$$

We conclude therefore from (4.24), (4.25) that

$$
-\partial F_{N} / \partial t|t=0 \geqq-\partial F / \partial t| t=0 .
$$


However the left side of (4.26) is just the left side of (4.17) and the right side of (4.26) is just the right side of (4.17). Q.E.D.

The strategy in the proof of Lemma 4.2 has been adopted from Lieb [12]. The proof of Theorem 1.1 now easily follows from Theorem 1.4 and the previous two lemmas.

\section{Appendix: Computation of Constants}

Here we compute the constants given in Theorem 1.1, Theorem 1.3 and Theorem 1.4. First we define the balls $B_{n, i}, 1 \leqq i \leqq \kappa(n), n=0, \pm 1, \ldots$. For each $x \in E_{n}$, let $\omega_{x}$ be the ball with center $x$ and radius $2^{-(n+3)}$. Let $\omega_{n, i}, 1 \leqq \mathrm{i} \leqq \kappa(\mathrm{n})$, be a maximal set of disjoint balls $\omega_{x}$. We define $B_{n, i}$ then as the ball concentric with $\omega_{n, i}$ but with radius $2^{-(n+1)}$. It is easy to see that the balls $B_{n, i}, 1 \leqq i \leqq \kappa(n)$, cover the neighbourhood of $E_{n}$ with radius $2^{-(n+2)}$. In fact by definition of the $\omega_{n, i}$ every point $x \in E_{n}$ is a distance smaller than $2^{-(n+2)}$ from the center of some $\omega_{n, i}$. Thus any point in the $2^{-(n+2)}$ neighbourhood of $E_{n}$ is a distance smaller than $2^{-(n+1)}$ from the center of some $\omega_{n, i}$. The point is therefore contained in $B_{n, i}$.

We compute the intersection number of the balls $B_{n, i}, 1 \leqq i \leqq \kappa(n)$. Suppose $x$ is in $r$ balls $B_{n, i}$. Then the $r$ disjoint balls $\omega_{n, i}$ are contained in the ball with center $x$ and radius $5 \cdot 2^{-(n+3)}$. It follows therefore that

$$
r \frac{4}{3} \pi\left[2^{-(n+3)}\right]^{3} \leqq \frac{4}{3} \pi\left[5 \cdot 2^{-(n+3)}\right]^{3} .
$$

Hence the intersection number of the balls $B_{n, i}, 1 \leqq i \leqq \kappa(n)$, is at most 125 . Now suppose $x \in \mathbb{R}^{3}$. Then $x \in E_{m}$ for some integer $m$. In view of (3.17) the point $x$ does not lie in any ball $B_{n, i}$ with $|n-m|>1$. Hence $x$ lies in at most $3 \cdot 125=375$ balls. Thus the intersection number of the balls $B_{n, i}, 1 \leqq i \leqq \kappa(n), n=0, \pm 1, \ldots$ is at most 375.

We consider Lemma 3.2. With the balls $B_{n, i}$ defined above we may take for $C_{0}$ and $\kappa$ the values

$$
C_{0}=8.45 \cdot 10^{17} ; \quad \kappa=7.5 \cdot 10^{6} .
$$

In Lemma 3.3 we obtain, with $C_{p, q}$ as in Lemma 1.2,

$$
C\left(N_{0}\right)=1.5 \cdot 10^{3} N_{0}^{2 / 3} C_{p, q}+1.7 \cdot 10^{7} N_{0}^{\frac{11}{3}}
$$

and the universal constant $\kappa$ in (3.26) is

$$
\kappa=1.1 \cdot 10^{14} .
$$

In Lemma 3.4 we take $\phi(x)$ be

$$
\phi(x)=G(10-64|x|),
$$

where $G(t)$ is the function defined by

$$
\begin{gathered}
G(t)=30 \int_{0}^{t} s^{2}(s-1)^{2} d s, \quad 0 \leqq t \leqq 1 \\
G(t)=0 \quad \text { if } \quad t<0, \quad G(t)=1 \quad \text { if } t>1 .
\end{gathered}
$$


For the function $\phi(x)$ and the lattice $\mathscr{L}$ defined in Lemma 3.4 we find the constant $C$ in Lemma 3.1 to be given by

$$
C=1.8 \cdot 10^{-6} \text {. }
$$

It is clear that the value of $\kappa$ we wish to take in the statement of Lemma 3.4 is a sum of the values of $\kappa$ given in (A.2) and (A.4). With this value of $\kappa$ the number $N_{0}$ turns out to be

$$
N_{0}=5.6 \cdot 10^{46} \text {. }
$$

The constant $C\left(N_{0}\right)$ in the statement of Lemma 3.4 is given by

$$
C\left(N_{0}\right)=1.5 \cdot 10^{27} N_{0}^{-1 / 3} \text {. }
$$

We may now compute the constants $C_{1}$ and $C_{2}$ in Theorem 1.3. With $N_{0}$ given by (A.8) we find from (A.3) that

$$
C_{1}=8.4 \cdot 10^{34} ; \quad C_{2}=3.7 \cdot 10^{161} .
$$

Next we compute the constants in Theorem 1.4. First we consider the inequalities (4.9) and (4.10). It is in fact sufficient to use only functions which are piecewise constant on the subcubes of $Q_{0}$. To compute the constant in (4.9) we need to construct a function similar to $G(t)$ in (A.6), but with four continuous derivatives. If we use a construction similar to $G(t)$ we obtain for $C$ in (4.9) a value

$$
C=1.8 \cdot 10^{-19} \text {. }
$$

The proof of (4.10) in [5] requires the use of the Muckenhoupt $A_{\infty}$ condition. Here we give a direct proof from which we can compute the constant in (4.10).

Lemma A.1. Let $Q$ be a cube in $\mathbb{R}^{3}$ and $u(x)$ be a function on $Q$ which is orthogonal to the constant function on $Q$. Then there is a universal constant $C$ such that

$$
\int_{Q} \frac{|u(x)|^{2}}{|x-y|} d x \leqq C \sum_{Q^{\prime} \subset Q}\left(\operatorname{diam} Q^{\prime}\right)^{-1}\left\|\hat{u}\left(Q^{\prime}\right)\right\|^{2} .
$$

Proof. We have

and therefore

$$
u=\sum_{Q^{\prime} \subset Q} \hat{u}\left(Q^{\prime}\right)
$$

$$
\int_{Q} \frac{|u(x)|^{2}}{|x-y|} d x=\sum_{Q^{\prime}} \int \frac{\left|\hat{u}\left(Q^{\prime}\right)\right|^{2}}{|x-y|} d x+2 \sum_{Q^{\prime} \supset Q^{\prime \prime}} \int \frac{\hat{u}\left(Q^{\prime}\right) \hat{u}\left(Q^{\prime \prime}\right)}{|x-y|} d x,
$$

where $Q^{\prime} \supset Q^{\prime \prime}$ means strict inclusion of $Q^{\prime \prime}$ in $Q^{\prime}$. For each subcube $Q^{\prime \prime}$ let $r_{Q^{\prime \prime}}$ be the distance from the center of $Q^{\prime \prime}$ to $y$. Evidently if $r_{Q^{\prime \prime}} \geqq\left(\operatorname{diam} Q^{\prime \prime}\right)$ then there is a universal constant $C$ such that

$$
|| x-\left.y\right|^{-1}-r_{Q^{\prime \prime}}^{-1} \mid \leqq C\left(\operatorname{diam} Q^{\prime \prime}\right) / r_{Q^{\prime \prime}}^{2}, \quad x \in Q^{\prime \prime} .
$$

We define sets $S_{k}, k=0,1, \ldots$ as follows:

$$
\begin{gathered}
S_{0}=\left\{Q^{\prime \prime}: r_{Q^{\prime \prime}}<\left(\operatorname{diam} Q^{\prime \prime}\right)\right\}, \\
S_{k}=\left\{Q^{\prime \prime}: 2^{k-1}\left(\operatorname{diam} Q^{\prime \prime}\right) \leqq r_{Q^{\prime \prime}}<2^{k}\left(\operatorname{diam} Q^{\prime \prime}\right)\right\}, \quad k \geqq 1 .
\end{gathered}
$$

Notice that we may find a constant $C$ such that for any integer $n$,

$$
\#\left\{Q^{\prime \prime} \in S_{k}: \operatorname{diam} Q^{\prime \prime}=2^{n}\right\} \leqq C 2^{3 k} \text {. }
$$


Next we write the double sum on the right in (A.14) as

$$
\sum_{Q^{\prime} \supset Q^{\prime \prime}}=\sum_{k=0}^{\infty} \sum_{\substack{Q^{\prime \prime} \in S_{k} \\ Q^{\prime \prime} \subset Q^{\prime}}}
$$

For $Q^{\prime \prime} \in S_{k}, Q^{\prime \prime} \subset Q^{\prime}$ it is easy to see from (A.15) and the orthogonality of $\hat{u}\left(Q^{\prime}\right)$ and $\hat{u}\left(Q^{\prime \prime}\right)$ that

$$
\left|\int \frac{\hat{u}\left(Q^{\prime}\right) \hat{u}\left(Q^{\prime \prime}\right)}{|x-y|} d x\right| \leqq C 2^{-2 k}\left(\operatorname{diam} Q^{\prime}\right)^{-3 / 2}\left(\operatorname{diam} Q^{\prime \prime}\right)^{1 / 2}\left\|\hat{u}\left(Q^{\prime}\right)\right\|\left\|\hat{u}\left(Q^{\prime \prime}\right)\right\| .
$$

Thus we have

$$
\begin{aligned}
\sum_{\substack{Q^{\prime \prime} \in S_{k} \\
Q^{\prime \prime} \subset Q^{\prime}}} \leqq & C 2^{-2 k} \sum\left(\operatorname{diam} Q^{\prime}\right)^{-3 / 2}\left(\operatorname{diam} Q^{\prime \prime}\right)^{1 / 2}\left\|\hat{u}\left(Q^{\prime}\right)\right\|\left\|\hat{u}\left(Q^{\prime \prime}\right)\right\| \\
\leqq & C 2^{-2 k}\left[\sum\left(\operatorname{diam} Q^{\prime \prime}\right)^{\varepsilon}\left(\operatorname{diam} Q^{\prime}\right)^{-1-\varepsilon}\left\|\hat{u}\left(Q^{\prime}\right)\right\|^{2}\right]^{1 / 2} \\
& \cdot\left[\sum\left(\operatorname{diam} Q^{\prime \prime}\right)^{1-\varepsilon}\left(\operatorname{diam} Q^{\prime}\right)^{-2+\varepsilon}\left\|\hat{u}\left(Q^{\prime \prime}\right)\right\|^{2}\right]^{1 / 2}
\end{aligned}
$$

by the Schwarz inequality, for any arbitrary $\varepsilon$.

It is clear from (A.17) that if we take $\varepsilon$ with $0<\varepsilon<2$ that the sum in (A.20) is bounded by

$$
C 2^{-k / 2} \sum\left(\operatorname{diam} Q^{\prime}\right)^{-1}\left\|\hat{u}\left(Q^{\prime}\right)\right\|^{2} .
$$

On summing (A.21) with respect to $k$ we obtain the result of the lemma. Q.E.D.

We may calculate easily a value for the number $C$ in (A.12) for which we obtain

$$
C=9000 \text {. }
$$

This is the constant we need for (4.10). We can now calculate the constants $C\left(N_{0}\right)$ and $\kappa$ in (4.2). In our calculation the coefficient of $\varrho_{1}(x)^{4 / 3}$ is a fourth power of $N_{0}$ while the coefficient of $\left\langle\varrho_{1}^{1 / 2}, H_{1} \varrho_{1}^{1 / 2}\right\rangle$ is linear in $N_{0}$. In view of the high value of $N_{0}$ we have we may take

$$
C\left(N_{0}\right)=3 \cdot 10^{9} N_{0}^{4},
$$

while the value of $\kappa$ may be taken as in (A.4). Then, taking $N_{0}$ as in (A.8) we obtain from (A.23) the value $C=10^{198}$ for the constant in Theorem 1.4. Since the constant in Daubechie's theorem is greater than $10^{-2}$ we may take $\alpha=10^{200}$ in Theorem 1.1.

Acknowledgements. I should especially like to thank Mark Ashbaugh for many hours of helpful discussion. Thanks are also due to Barry Simon for supplying the proof of the final lemma in the paper, John Lewis for bringing my attention to [8] of the bibliography, and Nigel Kalton for interesting conversation.

\section{References}

1. Daubechies, I.: An uncertainty principle for fermions with generalized kinetic energy. Commun. Math. Phys. 90, 511-520 (1983)

2. Daubechies, I., Lieb, E.: One electron relativistic molecules wuth Coulomb interaction. Commun. Math. Phys. 90, 497-510 (1983)

3. Dyson, F., Lenard, A.: Stability of matter. I. Jr. Math. Phys. 8, 423-434 (1967) 
4. Federbush, P.: A new approach to the stability of matter problem. II. Jr. Math. Phys. 16, 706-709 (1975)

5. Fefferman, C.: The uncertainty principle. Bull. Am. Math. Soc. 9, 129-206 (1983)

6. Hoffman-Ostenhof, M.T.: Schrödinger inequalities and asymptotic behaviour of the electron density of atoms and molecules. Phys. Rev. A 16, 1782-1785 (1977)

7. Lenard, A., Dyson, F.: Stability of matter. II. Jr. Math. Phys. 9, 698-711 (1968)

8. Lewis, J., Pulé, J., de Smedt, P.: The super-stability of pair potentials of positive type. Preprint (1983)

9. Lieb, E.: A lower bound for Coulomb energies. Phys. Lett. 70 A, 444-446 (1979)

10. Lieb, E.: The stability of matter. Rev. Mod. Phys. 48, 553-569 (1976)

11. Lieb, E.: Simplified approach to the ground state energy of an imperfect Bose gas. Phys. Rev. 130, 2518-2528 (1963)

12. Lieb, E.: Existence and uniqueness of the minimizing solution of Choquard's nonlinear equation. Stud. Appl. Myth. 57, 93-105 (1977)

13. Lieb, E., Narnhofer, H.: The thermodynamic limit for Jellium. J. Stat. Phys. 12, 291-310 (1975), Errata 14, 465 (1976)

14. Lieb, E., Oxford, S.: An improved lower bound on the indirect Coulomb energy. Int. J. Q. Chem. 19, 427-439 (1981)

15. Lieb, E., Thirring, W.: Gravitational collapse in quantum mechanics with relativistic kinetic energy. Ann. Phys. (to appear)

16. Lieb, E., Thirring, W.: A bound for the kinetic energy of Fermions which proves the stability of matter. Phys. Rev. Lett. 35, 687-689 (1975), Errata 35, 1116 (1975)

17. Thirring, W.: Quantum mechanics of large systems. Berlin, Heidelberg, New York: Springer 1983

Communicated by B. Simon

Received November 1, 1983; in revised form April 5, 1984 